\title{
Two-dimensional fluorescence resonance energy transfer as a probe for protein folding: A theoretical study
}

\author{
Christina L. Ting ${ }^{1, a)}$ and Dmitrii E. Makarov ${ }^{1,2, b)}$ \\ ${ }^{1}$ Department of Chemistry and Biochemistry, University of Texas at Austin, Austin, Texas 78712, USA \\ ${ }^{2}$ Institute for Theoretical Chemistry, University of Texas at Austin, Austin, Texas 78712, USA
}

(Received 29 October 2007; accepted 26 December 2007; published online 17 March 2008)

\begin{abstract}
We describe a two-dimensional (2D), four-color fluorescence resonance energy transfer (FRET) scheme, in which the conformational dynamics of a protein is followed by simultaneously observing the FRET signal from two different donor-acceptor pairs. For a general class of models that assume Markovian conformational dynamics, we relate the properties of the emission correlation functions to the rates of elementary kinetic steps in the model. We further use a toy folding model that treats proteins as chains with breakable cross-links to examine the relationship between the cooperativity of folding and FRET data and to establish what additional information about the folding dynamics can be gleaned from 2D, as opposed to one-dimensional FRET experiments. We finally discuss the potential advantages of the four-color FRET over the three-color FRET technique. (C) 2008 American Institute of Physics. [DOI: 10.1063/1.2835611]
\end{abstract}

\section{INTRODUCTION}

Single-molecule experiments are becoming increasingly popular as the preferred method for probing the kinetics of complex biomolecular phenomena. ${ }^{1-5}$ These techniques usually report the conformational state of an individual molecule with a single conformation-dependent variable. For example, in mechanical pulling experiments this conformationdependent variable is the distance between the points at which the molecule is attached to the pulling setup. In fluorescence resonance energy transfer (FRET) experiments, this variable is the emission from a dye molecule (the acceptor) that has been indirectly excited through FRET from another dye (the donor). The emission from the acceptor is strongly dependent on the donor-acceptor distance $r$, thus providing a spectroscopic ruler for measuring intramolecular distances. ${ }^{2,5-10}$ A convenient structural measure is the FRET efficiency $E$, which is related to $r$ through the equation

$$
E(r)=\frac{R_{0}^{6}}{r^{6}+R_{0}^{6}},
$$

where $R_{0}$ is the Förster radius.

Measuring the FRET efficiency $E(t)$ as a function of time may provide direct information about elementary steps of complex biochemical phenomena (see, e.g., Refs. 6 and 11-13). However, uncovering the underlying multidimensional dynamics of the system at hand from a onedimensional experimental time series such as $E(t)$ poses a problem that has challenged both theorists and experimentalists. ${ }^{14-32}$ Naturally, increasing the number of observables probed simultaneously in the same single-

\footnotetext{
${ }^{a}$ Present address: Biochemistry and Molecular Biophysics Option, California Institute of Technology, Pasadena, CA 91125.

b)Electronic mail: makarov@mail.cm.utexas.edu.
}

molecule experiment is viewed as a promising venue with the potential to make single-molecule studies more informative. ${ }^{1,33,34}$

Recently, multicolor FRET experiments have been reported $^{35-39}$ in which the emission from two acceptor molecules is simultaneously monitored, thus providing information about two intramolecular dimensions at the same time. The main goal of the present work is to illustrate-using computer simulations-what kind of information about the underlying single-molecule dynamics can be gleaned by simultaneously observing more than one conformational variable. We hope that our study will provide a theoretical framework for interpreting future experimental results for such two- (or multi) dimensional single-molecule studies.

Multidimensional single-molecule methods may be particularly useful for studies of protein and RNA folding. A number of single-molecule, in particular, single-molecule FRET, studies have already provided a wealth of information about various aspects of folding kinetics. ${ }^{1,8,10,11,40-48}$ For example, FRET photoemission statistics have recently been used to probe the ultrafast dynamics of protein collapse ${ }^{47}$ (also see Refs. 49 and 50 for the background). While these developments are impressive, we believe that multidimensional single-molecule experiments are poised to tackle the more ambitious issue concerning the mechanisms of protein folding.

The folding mechanism, i.e., the sequence of events through which an initially unstructured polypeptide chain attains its native conformation, is a long standing issue in biophysics. Many models of folding kinetics have been proposed, each postulating a different order of events and varied degrees of cooperativity for the formation of secondary structure and/or long range order. ${ }^{51-63}$ Although computer simulations can test some of these assumptions, direct experimental evidence for any specific folding scenario is hard to come by. 

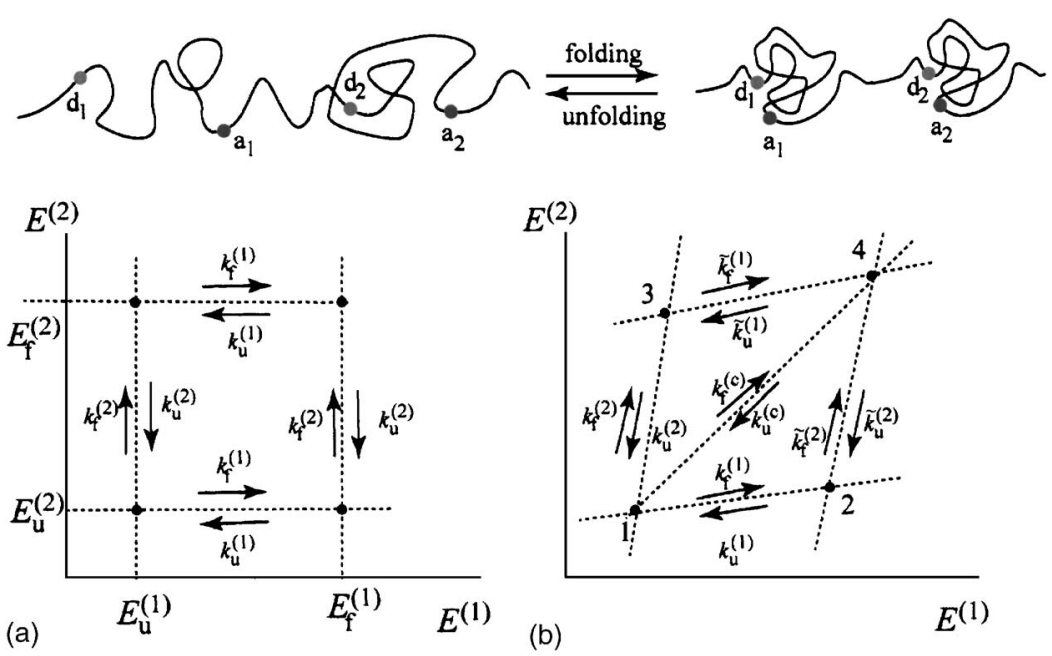

FIG. 1. Folding of a two-domain protein as manifested by $2 \mathrm{D}$ FRET, where each domain has been tagged with a donor-acceptor FRET pair. (A) For uncoupled domains, states of the protein form a rectangle in the $E^{(1)}-E^{(2)}$ plane. (B) Kinetic coupling between conformational dynamics of each domain alters the rate constants of folding and unfolding; structural coupling between two domains alters their FRET efficiencies such that the states no longer form a rectangle.

The inherent difficulty of using one-dimensional singlemolecule data to distinguish between cooperative and noncooperative folding scenarios is illustrated by the recent controversy regarding cooperativity in the folding of polyubiquitin domains under mechanical forces. ${ }^{64,65}$ Fernandez and $\mathrm{Li}^{64}$ studied the refolding of a multidomain polyubiquitin chain by monitoring the decrease in the overall extension of the chain. As was shown by subsequent theoretical studies, ${ }^{65,66}$ the dynamics of the chain extension is similar regardless of whether or not each domain folds independently. This makes differentiating between the cooperative and noncooperative scenarios difficult. On the other hand, if one could monitor the extensions of two individual domains, as in simulation studies, then resolving this issue would become trivial. It should further be possible to quantify the degree to which these events are cooperative. To do so, one needs to start with a definition of the "degree of cooperativity." In the literature, this term has been used in a somewhat loose sense to refer to many-body effects, ${ }^{67}$ specific folding pathways, ${ }^{53}$ sharpness of the folding transition, ${ }^{68}$ or the existence of a slow relaxation mode in the folding kinetics. ${ }^{69}$ We will use cooperativity in a narrower sense to refer to the mechanism through which the formation of partial native order speeds up subsequent folding events, thereby facilitating the global folding of the chain, see below.

More generally, we will talk about the dynamic and structural coupling between conformation-dependent variables. In the following sections we will introduce parameters that quantify this coupling. While it is possible to formulate our theory for any set of structural variables that characterize the dynamics of any macromolecular system, in order to be specific we are going to explore the relationship between the underlying folding dynamics of a protein and the statistics of its two-dimensional (2D) FRET trajectory $\left\{E^{(1)}(t), E^{(2)}(t)\right\}$, which can be recorded by simultaneously measuring the emission from two donor-acceptor pairs. This provides an experimental measure of correlation in protein dynamics. In practice, spectral overlap among dyes makes it difficult to design two completely independent donor-acceptor pairs. It is more feasible to have one donor and two acceptors, as has already been accomplished in the three-color FRET schemes of Refs. 37-39. This technique introduces additional cou- pling between $E^{(1)}$ and $E^{(2)}$ since the two acceptors must compete for the same excitation. The additional coupling through competing FRET pathways must be distinguished from the inherent dynamic coupling (if any) between the structural variables represented by $E^{(1)}$ and $E^{(2)}$. We will discuss how this can be accomplished in Sec. IV.

The rest of this paper is organized as follows. In Sec. II we will derive general expressions for the correlation functions of the form $C_{\alpha \beta}(t)=\left\langle E^{(\alpha)}(t) E^{(\beta)}(0)\right\rangle$ under the assumption that the system of interest undergoes a Markovian process. We will discuss how these correlation functions reflect the cooperativity in the dynamics of the system and illustrate our results with a simple example involving the folding of two weakly coupled protein domains. The theory presented in Sec. II is general and can be applied within the framework of any Markovian model of protein dynamics (see, e.g., Refs. 69-73), thus providing a link between experiments and protein folding models. In Sec. III we will present two case studies of the 2D FRET dynamics for the toy model that views proteins as Gaussian chains with breakable cross-links corresponding to native contacts. ${ }^{59,72,73}$ While far from providing a realistic model of protein dynamics, Gaussian chains with cross-links (GCCLs) exhibit many proteinlike properties. The GCCL model further allows for a sensible description of folding kinetics, as opposed to pseudokinetic schemes that use Metropolis Monte Carlo moves as elementary kinetic steps and do not consider the free energy barriers controlling the rate of each step. The GCCL model has been previously used to describe the relationship between protein topology and folding rates ${ }^{59,72,73}$ and to study the effect of protein topology on the mechanical resistance in singlemolecule pulling experiments. ${ }^{74,75}$ Finally, in Sec. IV we will conclude with a discussion of the implications of our work for experimental single-molecule FRET studies.

\section{THEORY}

\section{A. A simple example: Two weakly correlated FRET pairs}

To illustrate how 2D FRET can be used to probe cooperativity in protein folding, we begin with the simple example illustrated in Fig. 1. Suppose we have a protein that 
consists of two domains, each folding independently. The folding and unfolding rate constants for the domains are, respectively, $k_{f}^{(\alpha)}$ and $k_{u}^{(\alpha)}(\alpha=1,2$ for domains 1 and 2).

To find out whether or not the two domains fold independently, we place a donor-acceptor pair $\left\{d_{1}, a_{1}\right\}$ on the first domain and a different pair $\left\{d_{2}, a_{2}\right\}$ on the second domain. For each of the pairs, we measure its instantaneous FRET efficiency $E^{(\alpha)}$, which takes on a high value $E_{f}^{(\alpha)}$ in the folded state and a lower value $E_{u}^{(\alpha)}$ in the unfolded state. Thus our "experimental" data will consist of two time-dependent functions, $E^{(1)}(t)$ and $E^{(2)}(t)$, which we call the FRET trajectories. From this, we can also calculate the joint probability distribution function for the two efficiencies $P\left(E^{(1)}, E^{(2)}\right)$. For two independent domains, this probability is a product of the probability distribution of each individual pair:

$$
P\left(E^{(1)}, E^{(2)}\right)=P_{1}\left(E^{(1)}\right) P_{2}\left(E^{(2)}\right) .
$$

As a result of the statistical independence of the signal from the two pairs, the average of their product is equal to the product of their averages:

$$
\left\langle E^{(1)} E^{(2)}\right\rangle=\left\langle E^{(1)}\right\rangle\left\langle E^{(2)}\right\rangle .
$$

A convenient measure of the correlation between simultaneous values of $E^{(1)}$ and $E^{(2)}$ is the correlation coefficient given by

$$
\kappa_{12}=\frac{\left\langle E^{(1)} E^{(2)}\right\rangle-\left\langle E^{(1)}\right\rangle\left\langle E^{(2)}\right\rangle}{\sqrt{\left\langle\left(E^{(1)}-\left\langle E^{(1)}\right\rangle\right)^{2}\right\rangle\left\langle\left(E^{(2)}-\left\langle E^{(2)}\right\rangle\right)^{2}\right\rangle}} .
$$

In the case of our independent domains, there is no correlation and $\kappa_{12}=0$. In general, this parameter satisfies the inequality $-1 \leqslant \kappa_{12} \leqslant 1$ (Cauchy-Schwarz inequality) where $\kappa_{12}=-1$ corresponds to maximum anticorrelation and $\kappa_{12}$ $=1$ to maximum correlation.

We can also consider the time dependence of the correlation functions for our system. The autocorrelation function of the FRET efficiency that undergoes a Markovian, twostate process is given by ${ }^{76}$

$$
\begin{aligned}
C_{\alpha \alpha}(t) & \equiv\left\langle E^{(\alpha)}(t) E^{(\alpha)}(0)\right\rangle-\left\langle E^{(\alpha)}\right\rangle^{2} \\
& =\frac{k_{u}^{(\alpha)} k_{f}^{(\alpha)}}{\left(k_{u}^{(\alpha)}+k_{f}^{(\alpha)}\right)^{2}}\left(E_{u}^{(\alpha)}-E_{f}^{(\alpha)}\right)^{2} \exp \left[-\left(k_{u}^{(\alpha)}+k_{f}^{(\alpha)}\right) t\right] .
\end{aligned}
$$

Because of the statistical independence of the two pairs, the cross-correlation function

$$
C_{12}(t)=C_{21}(t)=\left\langle E^{(1)}(t) E^{(2)}(0)\right\rangle-\left\langle E^{(1)}\right\rangle\left\langle E^{(2)}\right\rangle
$$

will be identically zero in this case.

We now lift the assumption of statistical independence between the two domains and suppose that that they are coupled. The effect of coupling can be conveniently represented by plotting the states of the protein and the transitions among them in the $E^{(1)}-E^{(2)}$ plane, as shown in Fig. 1. Assuming that the coupling preserves Markovian dynamics, the case involving only four states may result in three different scenarios or any of their combinations.

(1) There can be a structural effect through which the folding or unfolding of one domain also affects the confor-

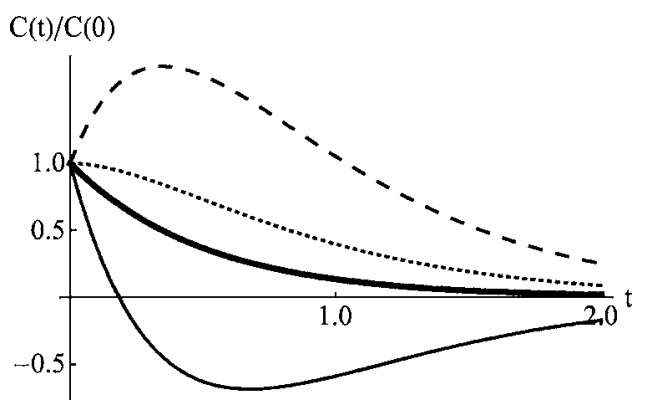

FIG. 2. Correlation functions for two coupled domains. The crosscorrelation function $C_{12}(t) / C_{12}(0)$ is plotted for $\xi=0.08, \Delta E=-0.01$ (thin solid line), $\xi=0.16, \Delta E=-0.01$ (dashed line), and $\xi=0.08, \Delta E=0$ (dotted line). See text for the definition of the coupling parameters. The heavy solid line shows the autocorrelation functions $C_{11}(t) / C_{11}(0)$ for all three cases, which are indistinguishable in this plot.

mation of the other, thereby altering the distantdependent FRET emission from both pairs in a single transition. Thus the points representing the states in the $E^{(1)}-E^{(2)}$ plane in Fig. 1(b) no longer form a rectangle as in Fig. 1(a).

(2) There can be a kinetic effect whereby the folding of a domain speeds up or slows down the folding of the other domain, so that $\widetilde{k}_{f}^{(1)} \neq k_{f}^{(1)}$ in Fig. 1(b). Similarly, $\widetilde{k}_{f}^{(2)} \neq k_{f}^{(2)}, \widetilde{k}_{u}^{(1)} \neq k_{u}^{(1)}, \widetilde{k}_{u}^{(2)} \neq k_{u}^{(2)}$. Note that the changes in these rate constants are not independent since they must preserve the detailed balance. In particular, one must have $\left(\widetilde{k}_{f}^{(2)} / \widetilde{k}_{u}^{(2)}\right)\left(k_{f}^{(1)} / k_{u}^{(1)}\right)=\left(k_{f}^{(2)} / k_{u}^{(2)}\right)\left(\widetilde{k}_{f}^{(1)} / \widetilde{k}_{u}^{(1)}\right)$.

(3) Finally, coupling can also introduce a new concerted pathway, in which both domains fold simultaneously. In Fig. 1(b), the folding and unfolding rate constants along this pathway are $\tilde{k}_{f}^{(c)}$ and $\tilde{k}_{u}^{(c)}$.

In Fig. 2, we illustrate how various combinations of scenarios 1 and 2 affect the time dependence of the crosscorrelation function $C_{12}(t)$. We have assumed that the folding of one domain speeds up the folding of the next, $\widetilde{k}_{f}^{(1)}=k_{f}^{(1)}(1+\xi)$ and $\widetilde{k}_{f}^{(2)}=k_{f}^{(2)}(1+\xi)$ for $\xi>0$, while the unfolding rate constant is left unchanged $\widetilde{k}_{u}^{(1)}=k_{u}^{(1)}, \widetilde{k}_{u}^{(2)}=k_{u}^{(2)}$. We set $k_{f}^{(1)}=k_{u}^{(1)}=k_{f}^{(2)}=k_{u}^{(2)}=1$. To account for the structural effect in scenario 1, we have assumed that the FRET efficiencies in the states labeled 1-4 in Fig. 1(b) are, respectively, given by

$$
\begin{aligned}
& \left(E^{(1)}, E^{(2)}\right)=\left(E_{u}^{(1)}, E_{u}^{(2)}\right), \quad\left(E_{f}^{(1)}-\Delta E, E_{u}^{(2)}+\Delta E\right), \\
& \left(E_{u}^{(1)}+\Delta E, E_{f}^{(2)}-\Delta E\right), \quad\left(E_{f}^{(1)}, E_{f}^{(2)}\right),
\end{aligned}
$$

where $\Delta E=0$ would correspond to Fig. 1(a).

In Fig. 2, we have chosen our coupling to be small $(\Delta E \ll 1, \xi \ll 1)$. As a result, it does not significantly affect the behavior of the autocorrelation function $C_{11}(t)$, which is essentially the same in all three cases shown in Fig. 2. However, the behavior of the cross-correlation function $C_{12}(t)$ is drastically affected by the choice of the coupling parameters $\xi$ and $\Delta E$. We also see that the time dependence of $C_{12}(t)$ can be nonmonotonic and strongly nonexponential. These features will be discussed in more detail in Sec. II B. Figure 2 
suggests that one can use the behavior of cross-correlation functions as a sensitive probe of the coupling between two conformational variables.

It is also instructive to consider the opposite extreme, where the coupling between the two domains is so strong that the folding kinetics is dominated by the concerted mechanism represented by the line connecting states 1 and 4 in Fig. 1(b). If we neglect all the rate constants except those for concerted folding and unfolding, $k_{f}^{(c)}$ and $k_{u}^{(c)}$, then $\kappa_{12}$ $=1$ (perfect correlation) and

$$
\begin{aligned}
C_{\alpha \beta}(t)= & \frac{k_{f}^{(c)} k_{u}^{(c)}}{\left(k_{f}^{(c)}+k_{u}^{(c)}\right)^{2}}\left(E_{u}^{(\alpha)}-E_{f}^{(\alpha)}\right)\left(E_{u}^{(\beta)}-E_{f}^{(\beta)}\right) \exp \left(-\left(k_{f}^{(c)}\right.\right. \\
& \left.\left.+k_{u}^{(c)}\right) t\right) .
\end{aligned}
$$

For this case, the correlation functions $C_{11}, C_{22}$, and $C_{12}$ have exactly the same time dependence and differ by only a factor. This is not surprising since, for a perfect two-state folder, we expect all observables to display the same time dependence. ${ }^{77}$

\section{B. 2D FRET statistics for the general case}

Consider now a more general situation, in which the dynamics of the system (e.g., the entire protein) can be described as a Markovian process involving $N$ states labeled $i$ $=1, \ldots, N$. For example, $N=4$ in Fig. 1 . The time dependence of the populations $p_{i}$ of these states is described by

$$
d p_{i} / d t=-\sum_{j \neq i} k_{i j} p_{i}+\sum_{j \neq i} k_{j i} p_{j}=\sum_{j} R_{i j} p_{j},
$$

where $k_{i j}$ is the rate constant for the transition from $i$ to $j$. We have introduced the rate matrix $\mathbf{R}$, whose off-diagonal elements are the rate constants, $R_{i j}=k_{j i}$, and whose diagonal elements are given by

$$
R_{i i}=-\sum_{j \neq i} R_{j i}
$$

We probe the system by monitoring two (or more) donoracceptor pairs, which we label with Greek letters $\alpha, \beta, \ldots$. With this notation, the FRET efficiency from the pair labeled $\alpha$ when the system is in state $i$ is $E_{i}^{(\alpha)}$.

We are interested in the correlation functions of the form

$$
C_{\alpha \beta}(t) \equiv\left\langle E^{(\alpha)}(t) E^{(\beta)}(0)\right\rangle-\left\langle E^{(\alpha)}\right\rangle\left\langle E^{(\beta)}\right\rangle .
$$

The conditional probability for finding the system at time $t$ in state $j$ provided it has started in state $i$ at $t=0$ is given by the element of the matrix $\left(e^{\mathbf{R} t}\right)_{i j}$. Using this fact, Eq. (10) can be written as

$$
C_{\alpha \beta}(t) \equiv E_{i}^{(\alpha)}\left(e^{\mathbf{R} t}\right)_{i j} p_{j}^{0} E_{j}^{(\beta)}-E_{i}^{(\alpha)} p_{i}^{0} p_{j}^{0} E_{j}^{(\beta)},
$$

where $p_{i}^{0}$,s are the equilibrium populations of the states. To simplify notation, from here on we have assumed summation over all double indices.

To obtain more insight into the time dependence represented by Eq. (11), we will examine its short- and long-time limits.

The short-time limit. To first order in time $t$, the matrix exponent can be approximated by

$$
e^{\mathbf{R} t} \approx 1+\mathbf{R} t .
$$

This gives

$$
\begin{aligned}
C_{\alpha \beta}(t) & \approx E_{i}^{(\alpha)}\left(1+R_{i j} t\right) p_{j}^{0} E_{j}^{(\beta)}-E_{i}^{(\alpha)} p_{i}^{0} p_{j}^{0} E_{j}^{(\beta)} \\
& =C_{\alpha \beta}(0)+t E_{i}^{(\alpha)} R_{i j} p_{j}^{0} E_{j}^{(\beta)} \\
& \equiv C_{\alpha \beta}(0)+t \eta_{\alpha \beta},
\end{aligned}
$$

where

$$
\eta_{\alpha \beta}=E_{i}^{(\alpha)} R_{i j} p_{j}^{0} E_{j}^{(\beta)} .
$$

Separating the off-diagonal elements of $\mathbf{R}$ from the diagonal ones in Eq. (14) and using Eq. (9) we have

$$
\begin{aligned}
\eta_{\alpha \beta} & =\sum_{i} E_{i}^{(\alpha)}\left[\sum_{j \neq i} R_{i j} p_{j}^{0} E_{j}^{(\beta)}+R_{i i} p_{i} E_{i}^{(\beta)}\right] \\
& =\sum_{i} E_{i}^{(\alpha)}\left[\sum_{j \neq i} R_{i j} p_{j}^{0} E_{j}^{(\beta)}-\sum_{j \neq i} R_{j i} p_{i}^{0} E_{i}^{(\beta)}\right] .
\end{aligned}
$$

The off-diagonal elements of the $\mathbf{R}$ matrix satisfy the detailed balance condition

$$
R_{i j} p_{j}^{0}=R_{j i} p_{i}^{0},
$$

so that we get

$$
\eta_{\alpha \beta}=\sum_{i} E_{i}^{(\alpha)}\left[\sum_{j \neq i} R_{i j} p_{j}^{0}\left(E_{j}^{(\beta)}-E_{i}^{(\beta)}\right)\right] .
$$

Now pick a pair of states $i$ and $j$. Their total contribution into the sum of Eq. (17) is

$$
\begin{aligned}
E_{i}^{(\alpha)} & R_{i j} p_{j}^{0}\left(E_{j}^{(\beta)}-E_{i}^{(\beta)}\right)+E_{j}^{(\alpha)} R_{j i} p_{i}^{0}\left(E_{i}^{(\beta)}-E_{j}^{(\beta)}\right) \\
& =E_{i}^{(\alpha)} R_{i j} p_{j}^{0}\left(E_{j}^{(\beta)}-E_{i}^{(\beta)}\right)-E_{j}^{(\alpha)} R_{i j} p_{j}^{0}\left(E_{j}^{(\beta)}-E_{i}^{(\beta)}\right) \\
& =\left(E_{i}^{(\alpha)}-E_{j}^{(\alpha)}\right) R_{i j} p_{j}^{0}\left(E_{j}^{(\beta)}-E_{i}^{(\beta)}\right),
\end{aligned}
$$

where Eq. (16) was used again. Thus we can rewrite Eq. (17) as

$$
\left.\eta_{\alpha \beta} \equiv \frac{d C_{\alpha \beta}}{d t}\right|_{t=0}=-\sum_{i<j}\left(E_{j}^{(\alpha)}-E_{i}^{(\alpha)}\right) R_{i j} p_{j}^{0}\left(E_{j}^{(\beta)}-E_{i}^{(\beta)}\right) .
$$

Equation (19) is one of the central results of this paper. Note that the physical meaning of the product $f_{i j}=R_{i j} p_{j}^{0}=k_{j i} p_{j}^{0}$ $=k_{i j} p_{i}^{0}$ is the equilibrium flux between states $i$ and $j$, which is the same in both directions because of the detailed balance.

Consider now the short-time behavior of a crosscorrelation function of the FRET efficiency for $\alpha \neq \beta$. Suppose that for any $i$ and $j$, each time a transition $i \rightarrow j$ occurs it affects at most one of the efficiencies, $E^{(\alpha)}$ or $E^{(\beta)}$, but not both. In the pictorial representation of Fig. 1, the jumps between states in the $E^{(\alpha)}-E^{(\beta)}$ plane take place in the direction of either axis. Then Eq. (19) gives $\eta_{\alpha \beta}=0$ and we say that there is no (measurable) structural coupling between the two pairs. This also means that the cross-correlation is a quadratic function of $t$ at short time, $C_{\alpha \beta}(t)=C_{\alpha \beta}(0)+\mu t^{2}$, unless the next term in the series happens to be zero as well.

To illustrate this, consider the two-domain case discussed in Sec. II A and illustrated in Figs. 1 and 2. If we make the additional assumption that $k_{f}^{(1)}=k_{f}^{(2)}=k_{f}$ and $k_{u}^{(1)}$ $=k_{u}^{(2)}=k_{u}$ then we find 


$$
\eta_{12}=\Delta E\left(2 \Delta E-\Delta E^{(1)}-\Delta E^{(2)}\right) \frac{k_{u} k_{f}\left(k_{u}+k_{f}+k_{f} \xi\right)}{k_{u}^{2}+2 k_{u} k_{f}+k_{f}^{2}(1+\xi)},
$$

where we have defined $\Delta E^{(1,2)}=E_{f}^{(1,2)}-E_{u}^{(1,2)}$. We see that $\eta_{12}$ becomes zero only if $\Delta E=0$ or $\Delta E=\left(\Delta E^{(1)}+\Delta E^{(2)}\right) / 2$. The case $\Delta E=0$ is illustrated by the dotted line in Fig. 2 and corresponds to pure scenario 2 above. It is straightforward to verify that the second case also corresponds to an arrangement where the four states depicted in Fig. 1 form a rectangle, although its edges are not necessarily aligned with $E^{(1)}$ and $E^{(2)}$.

When $\xi>0$ in our two-domain example, the cooperativity of folding transitions exists in the sense that folding of one domain facilitates the folding of the other. As a result, the parameter $\kappa_{12}$ [Eq. (4)] that measures correlations between simultaneous values of $E^{(1)}$ and $E^{(2)}$ is nonzero. We will call this kinetic cooperativity (or simply cooperativity) between the two domains as opposed to structural coupling, which we have quantified with the parameter $\eta_{\alpha \beta}$. Note that the term "kinetic cooperativity" may appear somewhat confusing because the parameter $\kappa_{\alpha \beta}$ can be obtained from the equilibrium distribution of the FRET efficiencies without any kinetics. This is because this distribution is related to the rates of the elementary transitions through the detailed balance.

The parameter $\kappa_{\alpha \beta}$ alone, however, cannot be used to distinguish structural coupling from kinetic cooperativity as it is influenced by both. For instance, using the same twodomain model, we find

$$
\begin{aligned}
\kappa_{12}= & \left(A_{1} A_{2}\right)^{-1 / 2}\left[k^{2} \Delta E\left(2 \Delta E-\Delta E^{(1)}-\Delta E^{(2)}\right)\right. \\
& \left.-k_{u} k_{f} \Delta E^{(1)} \Delta E^{(2)} \xi\right],
\end{aligned}
$$

where

$$
k^{2}=k_{u}^{2}+2 k_{u} k_{f}+k_{f}^{2}(1+\xi)
$$

and

$$
\begin{aligned}
A_{1,2}= & 2 \Delta E^{2} k^{2}-2 \Delta E^{(1,2)} \Delta E k^{2}+\left(\Delta E^{(1,2)}\right)^{2}\left(k_{u}+k_{f}\right)\left(k_{u}\right. \\
& \left.+k_{f}+k_{f} \xi\right) .
\end{aligned}
$$

In the absence of structural coupling (i.e., when $\Delta E=0) \kappa_{12}$ vanishes only if there is no cooperativity, $\xi=0$. However, in the general case $\kappa_{12}$ depends on both $\Delta E$ and $\xi$ and it may be nonzero even in the case of pure scenario $1(\xi=0)$. Moreover, according to Eq. (21) it can vanish when both $\Delta E$ and $\xi$ are finite if there is accidental cancellation between the structural and the kinetic effects. Finally, if there is no kinetic cooperativity (i.e., $\xi=0$ ) then we find from Eqs. (20) and (21) that both $\kappa_{12}$ and $\eta_{12}$ will vanish simultaneously for $\Delta E=0$ and $\Delta E=\left(\Delta E^{(1)}+\Delta E^{(2)}\right) / 2$.

The signs of the two parameters can be used to further understand the behavior of $C_{\alpha \beta}(t)$. For example, if both $\kappa_{\alpha \beta}$ and $\eta_{\alpha \beta}$ have the same sign then one can conclude that $C_{\alpha \beta}(t)$ has to be nonmonotonic (cf. the dashed line in Fig. 2). This is because $\kappa_{\alpha \beta}$ differs from $C_{\alpha \beta}(0)$ by only a positive constant [cf. Eqs. (4) and (6)], and any function $C_{\alpha \beta}(t)$ such that $\left.\left(d C_{\alpha \beta} / d t\right)\right|_{t=0}$ and $C_{\alpha \beta}(0)$ have the same sign and $C_{\alpha \beta}(\infty)=0$ must be nonmonotonic.
For autocorrelation functions, we have

$$
\eta_{\alpha \alpha}=-\sum_{i<j} R_{i j} p_{j}^{0}\left(E_{j}^{(\alpha)}-E_{i}^{(\alpha)}\right)^{2}<0 .
$$

This quantity is always negative. In our example of two weakly coupled domains, coupling has no effect on $\eta_{\alpha \alpha}$ in the zeroth order approximation. This is why $C_{11}(t)$ is monotonic and virtually the same in all three cases, as shown in Fig. 2.

The long time limit. At sufficiently long times, we generally expect both the autocorrelation functions and the cross-correlation functions to decay exponentially at the same rate, $C_{\alpha \beta}(t) \propto \exp \left(-\lambda_{1} t\right)$, where $\lambda_{1}$ is the lowest nonzero eigenvalue of the matrix $-\mathbf{R}$, except in special cases where the term proportional to $\exp (-\lambda t)$ is missing in the spectral decomposition of one of the correlation functions (cf. the case of two decoupled domains, where the two autocorrelations decay at different rates).

\section{FRET DYNAMICS IN THE GCCL MODEL}

In this section we will discuss 2D FRET in a model that has complex internal dynamics involving multiple conformations. This toy model, introduced in Refs. 72 and 73 and subsequently used to explore various aspects of protein folding and forced protein unfolding, ${ }^{59,74,75,78}$ considers the dynamics of Gaussian chains with breakable cross-links, each cross-link mimicking a native contact in a protein. Despite its naivety, this model displays a number of features observed in protein folding. Most notably, it offers a physical explanation of the correlations between folding rates and the native topology of single-domain proteins. ${ }^{59,72}$ Since the details of the model are given elsewhere, we will limit ourselves to a brief description and an explanation of how we have adapted the GCCL model to describe FRET.

\section{A. Description of the model}

Conformations of the chain. We will assume that our polypeptide chain consists of $L$ monomers connected by $L$ -1 bonds. In the GCCL model, the folded state of the chain is specified by the list of its $K$ native contacts $\left\{\left\{g_{1}, h_{1}\right\},\left\{g_{2}, h_{2}\right\}, \ldots,\left\{g_{K}, h_{K}\right\}\right\}$. In this notation, monomer $g_{1}$ is in contact with monomer $h_{1}$ and so on. Partially folded conformations will have some (but not all) of the native contacts formed. It is convenient to represent these states as binary strings of length $K$,

$$
i=C_{1} C_{2}, \ldots, C_{K} .
$$

Here $C_{k}=1$ if the contact between the monomers $g_{k}$ and $h_{k}$ is formed and $C_{k}=0$ otherwise. This allows us to label each conformation with a unique number $i$ obtained by converting the binary series back to base 10 . For example, the conformation involving $K=5$ native contacts, of which only the second contact $C_{2}$ is formed, is specified as $i=01000$. This uniquely sets $i=8$. The total number of states (i.e., binary strings) is $N=2^{K}$.

Stochastic dynamics of the chain. The model assumes that the formation or dissociation of each contact is described 
by first order kinetics. ${ }^{72,73}$ The rate constant for each contact to dissociate is given by

$$
k_{d}=\gamma e^{\varepsilon}
$$

where $\varepsilon$ is the free energy cost to form a contact measured in units of $k_{B} T$. Since all contacts in our model are binding, $\varepsilon$ is negative. We will further assume that this energy is the same for all native contacts. The preexponential $\gamma$ is given by $\gamma$ $=3 D / a^{2}$, where $D$ is the relative diffusion constant and $a$ is the distance used to determine when two monomers can form a native contact (see below). ${ }^{73}$ We set $\gamma=1$, which means that time is in units of $1 / \gamma$. Note that $k_{d}$ is independent of the state of the chain.

In contrast, the diffusion controlled rate constant $k_{f}$ for the formation of a native contact between the monomers $g$ and $h$ depends on which other contacts are already present. Assuming that the chain obeys Gaussian statistics and that a contact is formed between two monomers when the distance between them becomes less than $a$, this rate constant is given by $^{73,79}$

$$
\begin{aligned}
k_{f}\left[C_{1} C_{2}, \ldots, 0, \ldots, C_{K}\right. & \left.\rightarrow C_{1} C_{2}, \ldots, 1, \ldots, C_{K}\right] \\
& =\gamma(6 / \pi)^{1 / 2} a^{3} /\left\langle r_{g, h}^{2}\right\rangle^{3 / 2}
\end{aligned}
$$

where $\left\langle r_{g, h}^{2}\right\rangle$ is the mean square distance between $g$ and $h$. If no other contacts are present in the chain then this distance is given by

$$
\left\langle r_{g, h}^{2}\right\rangle=|g-h| b^{2}
$$

where $b$ is the effective length of the bond. As was shown in Ref. 73, Eq. (28) can be generalized for chains that contain arbitrary sets of contacts:

$$
\left\langle r_{g, h}^{2}\right\rangle=n_{\mathrm{eff}}\left(C_{1} C_{2}, \ldots, 0, \ldots, C_{K} ; g, h\right) b^{2},
$$

where $n_{\text {eff }}$ is the effective number of bonds between monomers $g$ and $h$ prior to the formation of a contact between them. The effective number of bonds depends on all other contacts that are already present and can be calculated using Kirchhoff's rules, as described in Refs. 73 and 78. Note that the rate constant of Eq. (27) depends only on the dimensionless ratio $a / b$. In what follows we set $(6 / \pi)^{1 / 2}(a / b)^{3}=0.5$.

Equations (27) and (29) introduce cooperativity into the model. Suppose, for example, that a native contact involves the chain ends. The formation of any other contact that creates an inner loop will effectively shorten the distance between the ends, thereby facilitating the subsequent collisions and therefore formation of the end-to-end native contact.

Description of FRET within the GCCL model. We now affix a FRET pair to our chain, with the donor residing on monomer $d$ and the acceptor on monomer $a$. The instantaneous efficiency of FRET between the donor and the acceptor separated by an instantaneous distance $r$ is given by Eq. (1). However, our model says nothing about the instantaneous value of $r$, nor can the instantaneous FRET efficiency be measured experimentally. Because $r$ typically fluctuates on time scales much faster than the time resolution of a FRET experiment, what is measured instead is the average FRET efficiency over a certain time window. ${ }^{41}$ As a result, the observable distribution of the FRET efficiency is nar- rower than one would expect on the basis of the true distribution of $r$ (the motional narrowing effect). Although in general the dependence on the window size may be quite complex, ${ }^{80}$ here we will assume that the time scale for the formation/dissociation of each native contact is slower than the time resolution of a FRET experiment. We will further assume that the time scale of the chain fluctuations while in any given state $i$ (as defined by the set of its formed native contacts) is faster than the time resolution. Thus our FRET "experiment" can resolve each of the individual $N=2^{K}$ states of the chain, for each yielding the value $E_{i}^{(\alpha)}=\langle E(r)\rangle_{i}$, which is the average of Eq. (1) over the probability distribution $p_{i}(r)$ of the donor-acceptor distance for that particular conformation. A recent study ${ }^{11}$ indicates that structural heterogeneity of the unfolded ensemble, as manifested by a broad distribution of FRET efficiencies, can indeed be resolved using single-molecule FRET.

Within our model, the probability distribution for the distance between the donor and the acceptor is Gaussian,

$$
p_{i}(r) d r=\left(\frac{3}{2 \pi n_{\mathrm{eff}} b^{2}}\right)^{3 / 2} e^{(-3 / 2)\left(r^{2} / b^{2} n_{\mathrm{eff}}\right)} 4 \pi r^{2} d r
$$

where $n_{\text {eff }}$ is the effective number of bonds between the donor and the acceptor in the given chain conformation [see Eq. (29)]. This gives, for the mean FRET efficiency in this conformation,

$$
\begin{aligned}
\left\langle E_{i}\right\rangle & =\int_{0}^{\infty} p_{i}(r) d r \frac{R_{0}^{6}}{R_{0}^{6}+r^{6}} \\
& =\int_{0}^{\infty} 4 \pi x^{2} d x\left(\frac{3}{2 \pi n_{\mathrm{eff}}}\right)^{3 / 2} e^{-(3 / 2)\left(x^{2} / n_{\mathrm{eff}}\right)} \frac{\widetilde{R}_{0}^{6}}{\widetilde{R}_{0}^{6}+x^{6}},
\end{aligned}
$$

where $\widetilde{R}_{0}=R_{0} / b$ is the dimensionless Förster radius. In all of our studies, we set $\widetilde{R}_{0}=4$.

We will now present two case studies using two different sets of native contacts. We will refer to these as structures 1 and 2, as shown in Fig. 3.

\section{B. Case study: Structure 1}

Structure 1, defined by the set $\{\{1,20\},\{10,30\},\{20,40\}$, $\{30,50\},\{40,60\},\{1,61\}\}$, has an interesting topology involving several pseudoknots. ${ }^{78}$ Both thermodynamically and kinetically, this structure shows features expected of small, single-domain proteins that fold via a two-state cooperative mechanism. ${ }^{81}$ To demonstrate how a single-molecule FRET experiment might reveal this mechanism, let us consider the case where the donor and the acceptor are attached to the ends of the chain, $\{d, a\}=\{1,61\}$. Figure 4 (top) shows the structure's denaturation curve defined as the average FRET efficiency (i.e., the average $\langle E\rangle=\sum_{i} p_{i}^{(0)} E_{i}$ ) plotted versus the contact energy $\varepsilon$ (measured in units of $k_{B} T$ ). The contact energy $\varepsilon$ measures the strength of the attractive interactions stabilizing the folded structure. When $\varepsilon$ is close to zero, the chain is denatured and the mean FRET efficiency is low. As one decreases the value of $\varepsilon$, the structure undergoes a "folding transition," which is manifested by the mean FRET effi- 

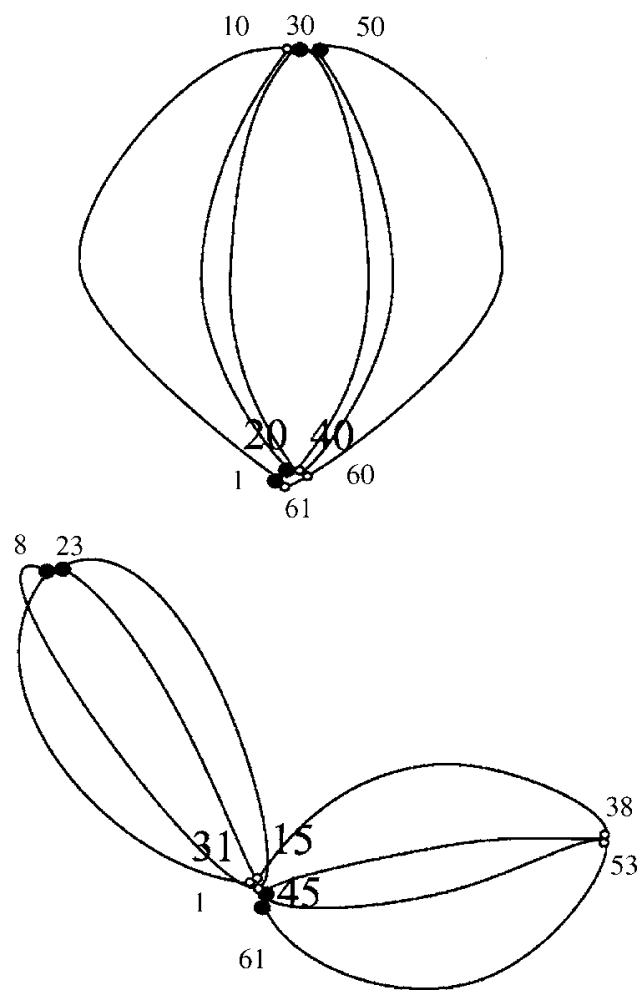

FIG. 3. A cartoon depiction of structure 1 (top) and structure 2 (bottom). Native contact points are shown as open circles. FRET donor and acceptor dyes are shown as filled circles.

ciency approaching the maximum value of 1 . Here, the donor-acceptor pair $\{1,61\}$ has formed and the polypeptide chain is in the folded conformation.

At this point the reader could raise the valid objection

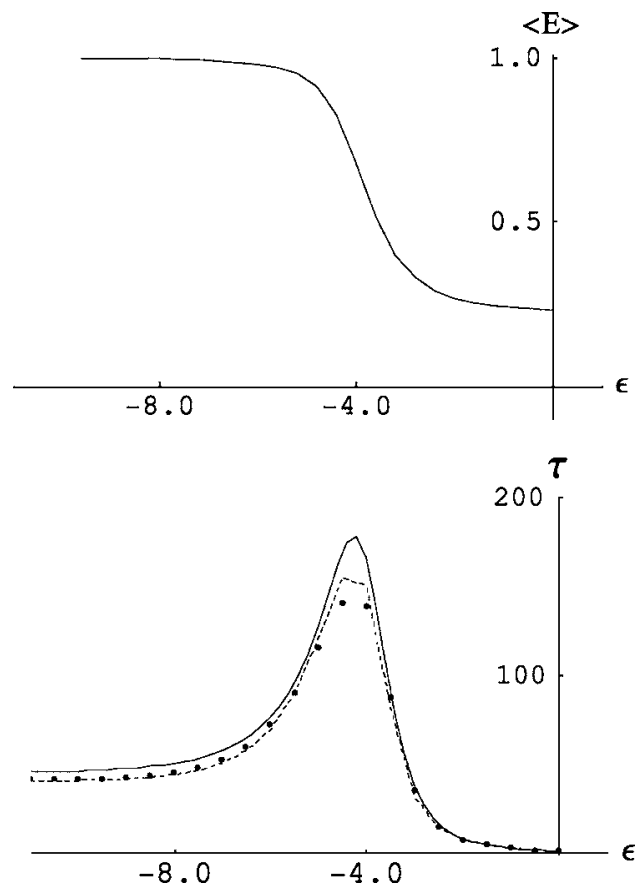

FIG. 4. Top: The average FRET efficiency $\langle E\rangle$ as a function of $\varepsilon$ for the donor-acceptor pair $\{1,61\}$ of structure 1. Bottom: The characteristic relaxation times $\tau_{1}$ (points), $\tau_{2}$ (dashed line), and $\tau_{3}$ (solid line) as a function of $\varepsilon$ for structure 1. See Eqs. (32)-(34) for the definition of these times. that the formation of one particular native contact $(\{1,61\})$ does not necessarily indicate the formation of the folded state consisting of all six native contacts. In fact, it is more common to use a collective coordinate, such as the fraction of the native contacts formed, to characterize the folding progress. However, it turns out that for this particular structure, the denaturation curve is similar regardless of the choice of the donor-acceptor pair as long as it coincides with a native contact. In other words, the donor-acceptor distances for different native monomer pairs are highly correlated, just as expected for a good "two-state folder." 81 2D FRET is well suited for probing this kind of correlation and we will return to this issue at the end of this section.

We now turn to the dynamics of our model. We are interested in defining a characteristic time scale $\tau$ that describes conformational rearrangements as observed by FRET. A natural way to do this is to consider the autocorrelation function of the FRET efficiency defined by Eq. (10). If the autocorrelation function decays approximately exponentially according to $C_{\alpha \alpha}(t)=C_{\alpha \alpha}(0) e^{-t / \tau}$ (as one expects for a perfect two-state folding protein ${ }^{69}$ ), then $\tau$ can be estimated by

$$
\tau_{1}=\int_{t=0}^{\infty} C_{\alpha \alpha}(t) d t / C_{\alpha \alpha}(0)
$$

or

$$
\tau_{2}=\int_{t=0}^{\infty} t C_{\alpha \alpha}(t) d t / \int_{t=0}^{\infty} C_{\alpha \alpha}(t) d t .
$$

On the other hand, using the spectral decomposition of Eq. (11), one finds that at long enough time the correlation function should decay as $C_{\alpha \alpha}(t) \propto e^{-\lambda_{1} t}$, where $\lambda_{1}$ is the lowest nonzero eigenvalue of the rate matrix $-\mathbf{R}$. This means that in the case of purely exponentially decaying correlation function, we should have

$$
\tau \equiv \tau_{3}=\frac{1}{\lambda_{1}} .
$$

In the case where the correlation function is nonexponential, all three times would be different. Equation (32) could, for example, be pathological if $C_{\alpha \alpha}(0)=0$, which is a common case for quantum mechanical emission autocorrelation functions that show photon antibunching behavior (see, e.g., Ref. 50). While this is not the case here, we can use the differences among $\tau_{1}, \tau_{2}$, and $\tau_{3}$ to assess deviations from nonexponential behavior.

In Fig. 4 (bottom), we plot $\tau_{1}, \tau_{2}$, and $\tau_{3}$ as a function of $\varepsilon$. We find that the differences between the three times are small, indicating that the behavior of the autocorrelation function is close to exponential. We further find that the correlation time $\tau$ exhibits a maximum as a function of $\varepsilon$ located near the midpoint of the denaturation curve. This is the typical chevron-plot type of behavior, ${ }^{82}$ which is commonly observed for two-state folding proteins. In terms of a simple two-state model, in which the rate constants for folding and unfolding are, respectively, $k_{f}(\varepsilon)$ and $k_{u}(\varepsilon)$, the correlation time is equal to $\tau=1 /\left[k_{u}(\varepsilon)+k_{f}(\varepsilon)\right]$ [cf. Eq. (7)]. As $k_{f}(\varepsilon)$ decreases and $k_{u}(\varepsilon)$ increases with increasing $\varepsilon, \tau$ exhibits a 
peak. We note that in our model this effective two-state behavior emerges from rather complex multistate kinetics of the model as a result of strong cooperativity among different contacts, see below.

We have also studied random contact sets and found that some of them show a sharp maximum of the relaxation time and a sharp denaturation transition while others do not. Consistent with earlier theoretical predictions, ${ }^{68,82}$ the latter sets do not show two-state behavior (i.e., a nearly exponential decay and a high degree of correlation among all contactssee below).

We now proceed to the case of 2D FRET and assume that two FRET pairs are attached to our chain, with the positions $\left\{d_{1}, a_{1}\right\}=\{1,20\}$ and $\left\{d_{2}, a_{2}\right\}=\{30,50\}$. Since the locations of these pairs coincide with native contacts, the two FRET signals probe whether or not the contacts are formed. Experimentally, one can measure the joint probability distribution of the two FRET efficiencies $P\left(E^{(1)}, E^{(2)}\right)$. Within our model (see Sec. II B), this is given by

$$
P\left(E^{(1)}, E^{(2)}\right)=\sum_{i} p_{i}^{(0)} \delta\left(E-E_{i}^{(1)}\right) \delta\left(E-E_{i}^{(2)}\right),
$$

which is a collection of $\delta$ peaks. Of course, in practice, these peaks are broadened by noise. In addition, incomplete motional narrowing may also contribute to the observable width. $^{41,42}$ To mimic these effects in our model, we simply replace each $\delta$ peak in Eq. (35) by a two-dimensional Gaussian of width $\Delta$, such that our joint probability distribution becomes

$$
P\left(E^{(1)}, E^{(2)}\right)=\sum_{i} p_{i}^{(0)} g\left(E-E_{i}^{(1)}\right) g\left(E-E_{i}^{(2)}\right)
$$

where

$$
g(E)=\frac{1}{\sqrt{2 \pi \Delta^{2}}} \exp \left(-\frac{E^{2}}{2 \Delta^{2}}\right) .
$$

We use the value $\Delta=0.05$ throughout. As a result, not all the distinct states can be resolved. Note that Eq. (36) preserves normalization of the joint probability density but may permit unphysical values of the FRET efficiency (e.g., $E>1$ ).

In Fig. 5, we plot $P\left(E^{(1)}, E^{(2)}\right)$ at $\varepsilon=-4$, corresponding to the midpoint of the denaturation curve. In addition, we also plot the discrete points $\left(E_{i}^{(1)}, E_{i}^{(2)}\right)$ corresponding to the distinct chain conformations [i.e., the locations of the $\delta$ peaks of Eq. (35)]. Finally, we show the connectivity of our model by plotting straight lines between any two conformations that are connected kinetically (i.e., there is a transition between them). In our model, only the elementary transitions that break a single contact or form a new contact are allowed.

Figure 5 shows similarities to both Figs. 1(a) and 1(b). The distribution $P\left(E^{(1)}, E^{(2)}\right)$ has four maxima corresponding to the cases where both contacts are formed, both contacts are unformed, and one contact formed and one unformed. These four peaks form a rectangle as in Fig. 1(a). It is possible to go from the peak where both contacts are broken to the peak where both contacts are formed along the edges of the rectangle. This corresponds to a pathway in which there is no structural coupling between the distances reported by $E^{(1)}$ and $E^{(2)}$ during each elementary transition. Another ex-

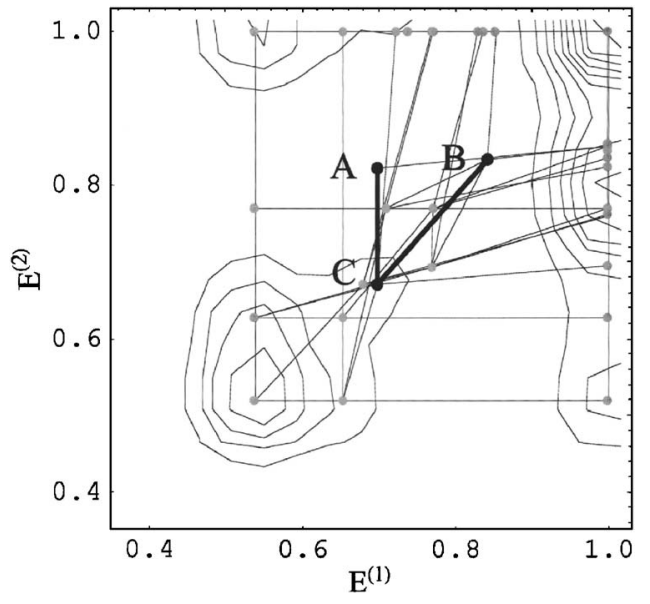

FIG. 5. Contour plot of the joint probability distribution of the FRET efficiency $P\left(E^{(1)}, E^{(2)}\right)$ at $\varepsilon=-4$ for structure 1 . The FRET pairs are defined by $\left\{d_{1}, a_{1}\right\}=\{1,20\}$ and $\left\{d_{2}, a_{2}\right\}=\{30,50\}$. Also shown are the points $\left(E_{i}^{(1)}, E_{i}^{(2)}\right)$ for each state $i$ and straight lines showing the connectivity of all possible paths. The lines $\mathrm{A} \rightarrow \mathrm{B}$ and $\mathrm{A} \rightarrow \mathrm{C}$ provide provide examples of elementary transitions with and without structural coupling between the FRET pairs, respectively (see Fig. 6).

ample of a transition that does not introduce structural coupling is illustrated in Fig. 6, in which we show the breaking of contact $\{40,60\}$, corresponding to line A-C in Fig. 5. This event affects $E^{(2)}$ without changing the value of $E^{(1)}$.

In addition to the above four peaks, intermediate peaks are also observed. Note the set of transitions aligned along the diagonal of the graph. These are the transitions showing structural coupling between the FRET pairs. For example, in the second transition shown in Fig. 6 (and shown as line B-C in Fig. 5), contact $\{1,61\}$ is broken. Intuitively, we can understand how this global event involving end monomers will affect both $E^{(1)}$ and $E^{(2)}$. (a)
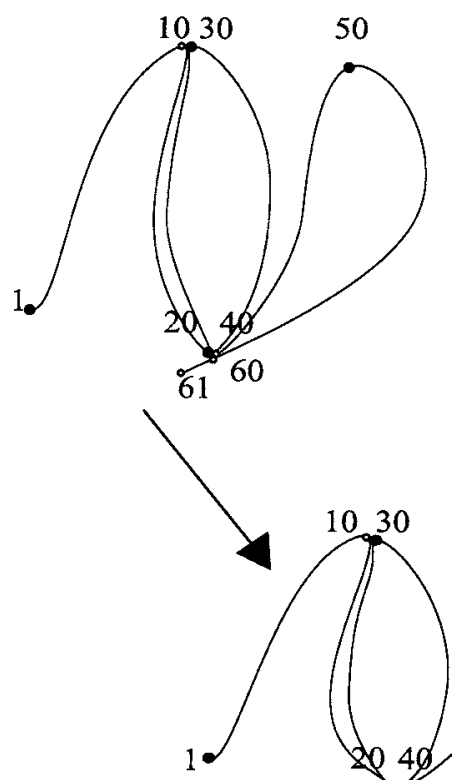

(b)

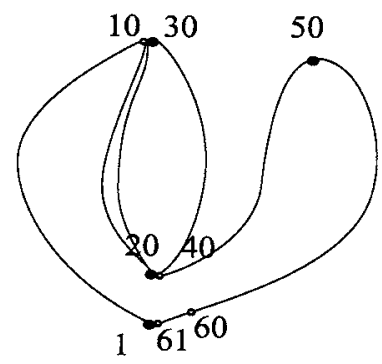

FIG. 6. A cartoon showing the rearrangements of the chain in the transition $\mathrm{A} \rightarrow \mathrm{C}$ and $\mathrm{B} \rightarrow \mathrm{C}$ highlighted in Fig. 5, with dye molecules shown as filled circles. 

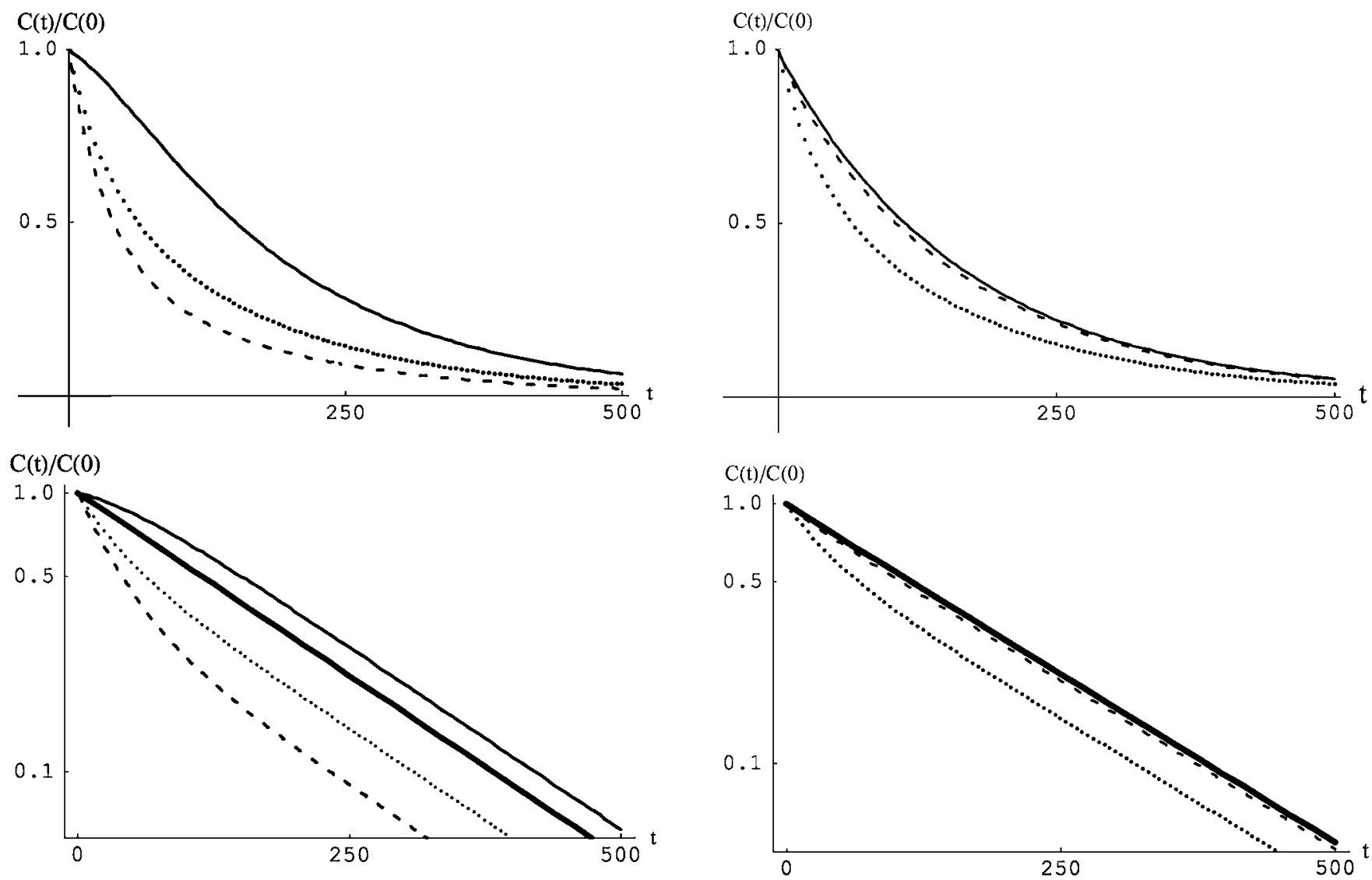

FIG. 7. Top: The correlation functions $C_{12}(t) / C_{12}(0)$ (solid line), $C_{11}(t) / C_{11}(0)$ (dotted line), and $C_{22}(t) / C_{22}(0)$ (dashed line) plotted for structure 1 at $\varepsilon=-4$. The FRET pairs are defined by $\left\{d_{1}, a_{1}\right\}=\{1,20\}$ and $\left\{d_{2}, a_{2}\right\}=\{30,50\}$. Bottom: Same correlation functions plotted on a semilogarithmic scale. The heavy solid line is $e^{-\lambda_{1} t}$ plotted vs $t$, where $\lambda_{1}$ is the lowest nonzero eigenvalue of the matrix $-\mathbf{R}$.

Because structural coupling between two pairs is seen only for some elementary transitions, it is not surprising that our choice of FRET pairs for structure 1 corresponds to an intermediate situation between having two independent contacts and two strongly correlated ones. As discussed in Sec. II, the degree of structural coupling should be reflected in the temporal behavior of the correlation functions of the FRET efficiencies. These correlation functions for structure 1 are shown in Fig. 7. While at long time they all decay exponentially at the same rate corresponding to the lowest eigenvalue of the rate matrix $-\mathbf{R}$ (bottom of Fig. 7, heavy solid line), at shorter times $C_{11}(t), C_{12}(t)$, and $C_{22}(t)$ behave differently. This, of course, indicates that our structure 1 is not a perfect two-state, cooperative folder, in which all native contacts form in a concerted fashion.

FIG. 8. Top: The correlation functions $C_{12}(t) / C_{12}(0)$ (solid line), $C_{11}(t) / C_{11}(0)$ (dotted line), and $C_{22}(t) / C_{22}(0)$ (dashed line) plotted for structure 1 at $\varepsilon=-4$. The FRET pairs are defined by $\left\{d_{1}, a_{1}\right\}=\{40,60\}$ and $\left\{d_{2}, a_{2}\right\}=\{1,61\}$. Bottom: Same correlation functions plotted on a semilogarithmic scale. The heavy solid line is $e^{-\lambda_{1} t}$ plotted vs $t$, where $\lambda_{1}$ is the lowest nonzero eigenvalue of the matrix $-\mathbf{R}$. Note the the cross-correlation function is indistinguishable from $e^{-\lambda_{1} t}$.

The degree of coupling differs for different pairs of contacts. If, for example, we select $\{40,60\}$ and $\{1,61\}$ as the locations of our two FRET pairs, then we find $C_{11}(t), C_{12}(t)$, and $C_{22}(t)$ (Fig. 8) to have considerably more similarity in their behavior. This finding is also consistent with the difference in the values of the correlation coefficient $\kappa_{12}$ [as defined in Eq. (4)] computed for these two choices of FRET pairs. Table I gives the values of the correlation coefficient for every possible choice of two FRET pairs (assuming that a FRET pair corresponds to a native contact). According to this table, our first choice of two contacts corresponds to the lowest correlation case $\left(\kappa_{12}=0.403\right)$ while the second choice corresponds to the highest correlation case $\left(\kappa_{12}=0.803\right)$. We

TABLE I. The correlation coefficients $\kappa_{12}$ for structure 1 , where $E^{(1)}$ and $E^{(2)}$ are for FRET pairs attached to the monomers of the corresponding row and column contacts of the table element.

\begin{tabular}{|c|c|c|c|c|c|c|}
\hline & $C_{1}$ & $C_{2}$ & $C_{3}$ & $C_{4}$ & $C_{5}$ & $C_{6}$ \\
\hline$C_{1}$ & & 0.634 & 0.567 & 0.403 & 0.529 & 0.783 \\
\hline$C_{2}$ & & & 0.635 & 0.434 & 0.414 & 0.652 \\
\hline$C_{3}$ & & & & 0.630 & 0.572 & 0.767 \\
\hline$C_{4}$ & & & & & 0.618 & 0.649 \\
\hline$C_{5}$ & & & & & & 0.803 \\
\hline$C_{6}$ & & & & & & \\
\hline
\end{tabular}


TABLE II. $\eta_{12}$ for structure 1 , where $E^{(1)}$ and $E^{(2)}$ are for FRET pairs attached to the monomers of the corresponding row and column contacts of the table element.

\begin{tabular}{lcccccc}
\hline \hline & $C_{1}$ & $C_{2}$ & $C_{3}$ & $C_{4}$ & $C_{5}$ & $C_{6}$ \\
\hline$C_{1}$ & $-6.2 \times 10^{-4}$ & $-2.3 \times 10^{-4}$ & $-8.0 \times 10^{-5}$ & $-3.4 \times 10^{-5}$ & $-4.5 \times 10^{-5}$ & $-3.5 \times 10^{-4}$ \\
$C_{2}$ & & $-7.1 \times 10^{-4}$ & $-2.3 \times 10^{-4}$ & $-1.2 \times 10^{-4}$ & $-3.6 \times 10^{-5}$ & $-3.1 \times 10^{-4}$ \\
$C_{3}$ & & & $-6.1 \times 10^{-4}$ & $-2.3 \times 10^{-4}$ & $-8.1 \times 10^{-5}$ & $-3.2 \times 10^{-4}$ \\
$C_{4}$ & & & & $-7.2 \times 10^{-4}$ & $-2.2 \times 10^{-4}$ & $-3.1 \times 10^{-4}$ \\
$C_{5}$ & & & & $-6.5 \times 10^{-4}$ & $-3.8 \times 10^{-4}$ \\
$C_{6}$ & & & & & $-9.4 \times 10^{-4}$ \\
\hline \hline
\end{tabular}

also note that for all possible choices of the two donoracceptor pairs, there is a significant correlation $(>0.4)$, consistent with the notion that the folding of structure 1 shows significant degree of cooperativity.

In Table II we give the values of $\eta_{12}=\left.\left(d C_{12} / d t\right)\right|_{t=0}$ for different choices of the two donor-acceptor pairs. As discussed in Sec. II, $\eta_{12}$ provides a direct measure of structural coupling between two FRET signals. Comparing Tables I and II, one observes a correlation between the values of $\kappa_{12}$ and of the structural coupling parameter $\eta_{12}$. For example, the least correlated donor-acceptor pairs $C_{1}$ and $C_{4}$ also correspond to the minimum value of $\left|\eta_{12}\right|$, while the most correlated pairs, $C_{5}$ and $C_{6}$, also result in the maximum value of $\left|\eta_{12}\right|$. However, for $C_{2}$ and $C_{4}$ we find a low value of $\kappa_{12}$ comparable to the pair $C_{1}$ and $C_{4}$ while the value $\left|\eta_{12}\right|$ is much higher.

In the GCCL model, the formation of a native contact reduces (or leaves unchanged) the effective number of bonds $n_{\text {eff }}$ between any pair of monomers. This means that the FRET efficiency from any pair of monomers should increase (or remain the same) upon the formation of any contact. Therefore every nonzero term in Eq. (19) is negative, since the change in both FRET efficiencies has the same sign, and $\eta_{12}$ will always be negative for our model. Because the rate of contact formation also increases with decreasing $n_{\mathrm{eff}}$, the GCCL model is frustration-free. Thus kinetic cooperativity

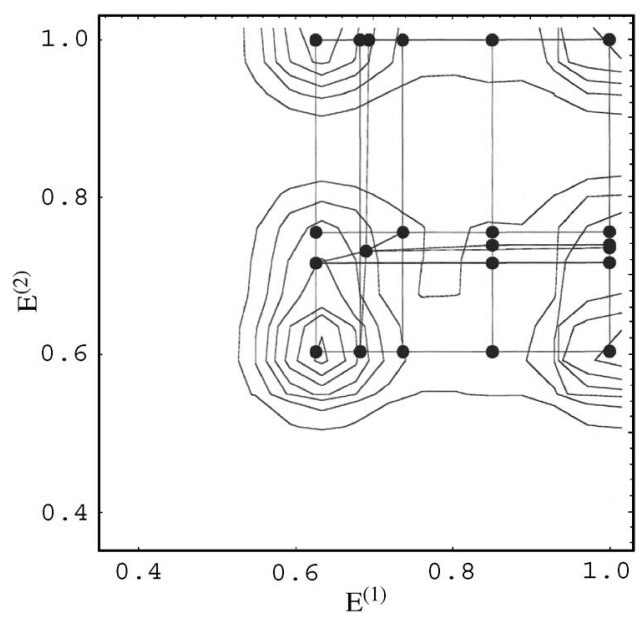

FIG. 9. Contour plot of the joint probability distribution of the FRET efficiency $P\left(E^{(1)}, E^{(2)}\right)$ at $\varepsilon=-4$ for structure 2. The FRET pairs are defined by $\left\{d_{1}, a_{1}\right\}=\{8,23\}$ and $\left\{d_{2}, a_{2}\right\}=\{45,61\}$. Also shown are the points $\left(E_{i}^{(1)}, E_{i}^{(2)}\right)$ for each state $i$ and straight lines showing the connectivity of all possible paths. of our model has essentially the same physical origin as the structural coupling observed through the FRET signals. This explains why $\left|\eta_{12}\right|$ and $\kappa_{12}$ behave similarly.

\section{Case study: Structure 2}

We now turn to structure 2 (Fig. 3), which consists of the native contacts specified by $\{\{1,31\},\{8,23\},\{31,45\},\{45,61\}$, $\{38,53\},\{15,45\}\}$. Without the presence of contact $\{15,45\}$, the chain segment between monomers 1 and 31 would be statistically independent from the segment between 31 and 61 . We can think of structure 2 as consisting of two domains coupled by $\{15,45\}$.
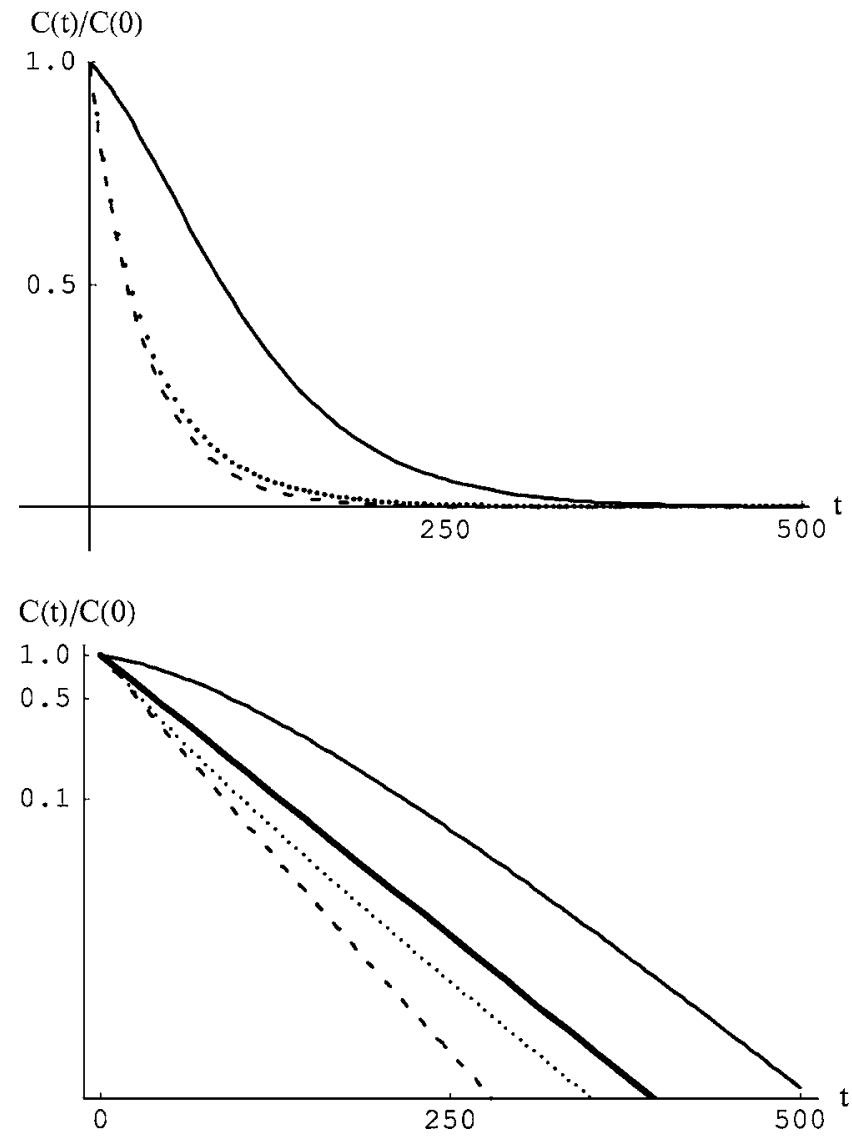

FIG. 10. Top: The correlation functions $C_{12}(t) / C_{12}(0)$ (solid line), $C_{11}(t) / C_{11}(0)$ (dotted line), and $C_{22}(t) / C_{22}(0)$ (dashed line) plotted for structure 2 at $\varepsilon=-4$. The FRET pairs defined by $\left\{d_{1}, a_{1}\right\}=\{8,23\}$ and $\left\{d_{2}, a_{2}\right\}$ $=\{45,61\}$. Bottom: Same correlation functions plotted on a semilogarithmic scale. The heavy solid line is $e^{-\lambda_{1} t}$ plotted vs $t$, where $\lambda_{1}$ is the lowest nonzero eigenvalue of the matrix $-\mathbf{R}$. 
TABLE III. The correlation coefficients $\kappa_{12}$ for structure 2, where $E^{(1)}$ and $E^{(2)}$ are for FRET pairs attached to the monomers of the corresponding row and column contacts of the table element.

\begin{tabular}{|c|c|c|c|c|c|c|}
\hline & $C_{1}$ & $C_{2}$ & $C_{3}$ & $C_{4}$ & $C_{5}$ & $C_{6}$ \\
\hline$C_{1}$ & & 0.763 & 0.218 & 0.017 & 0.063 & 0.538 \\
\hline$C_{2}$ & & & 0.128 & 0.010 & 0.036 & 0.366 \\
\hline$C_{3}$ & & & & 0.139 & 0.442 & 0.770 \\
\hline$C_{4}$ & & & & & 0.554 & 0.260 \\
\hline$C_{5}$ & & & & & & 0.260 \\
\hline$C_{6}$ & & & & & & \\
\hline
\end{tabular}

The FRET pairs $\left\{d_{1}, a_{1}\right\}=\{8,23\}$ and $\left\{d_{2}, a_{2}\right\}=\{45,61\}$ have been selected to probe the formation of a loop within each domain. In the absence of the contact $\{15,45\}$, there is no coupling between the two FRET pairs. Once the contact $\{15,45\}$ forms, it connects the two domains and induces a correlation between $E^{(1)}$ and $E^{(2)}$. This is seen in Fig. 9, where discrete states are shown in the $E^{(1)}-E^{(2)}$ plane, along with their connectivity. We observe a weak correlation in that only a small number of elementary transitions are observed to introduce structural coupling between $E^{(1)}$ and $E^{(2)}$. For most of the transitions the two FRET efficiencies change independently. The situation is thus similar to that discussed in the example of Sec. II A involving two independent domains.

The weak coupling between the two domains is reflected in the behavior of the correlation functions, see Fig. 10. Specifically, $C_{12}(t)$ exhibits strong nonexponential behavior and decays much slower than the autocorrelation functions $C_{11}(t)$ and $C_{22}(t)$. As discussed in Sec. II, slow initial decay of $C_{12}(t)$ (i.e., small absolute value of $\eta_{12}$ ) indicates weak structural coupling between the pairs, which is indeed observed in Fig. 9 and Table IV. The autocorrelation functions $C_{11}(t)$ and $C_{22}(t)$ appear to decay exponentially but each at a different rate, as seen from the semilogarithmic plot in Fig. 10. This is because the two domains are only weakly coupled and so each has its own folding/unfolding kinetics. At long time, one expects all of the correlation functions to decay as $\exp \left(-\lambda_{1} t\right)$, where $\lambda_{1}$ is the lowest nonzero eigenvalue of the rate matrix $-\mathbf{R}$. Examining Fig. 10, one may conclude that this is true for $C_{11}(t)$ but not for $C_{22}(t)$, which seemingly decays at a different rate. However, if we plotted these functions for much longer times, we would discover that the expected behavior, $C_{22}(t) \sim \exp \left(-\lambda_{1} t\right)$, is eventually recovered (data not shown). Since all of the correlation functions are essentially zero at times so long, recovering the correct exponential tail for $C_{22}(t)$ would be impractical experimentally.
These observations emphasize the potential value of measuring cross-correlation between FRET signals achieved in 2D FRET. In a conventional one-dimensional FRET experiment, one could separately measure $C_{11}(t)$ and $C_{22}(t)$, find them to decay at different rates, and erroneously conclude that the two domains are independent. This is because the correct exponential tails are hard to measure. On the other hand, even the short-time behavior of $C_{12}(t)$ would immediately indicate the degree of coupling between the two domains.

Examining the correlation coefficient $\kappa_{12}$ for all possible choices of the two FRET pairs corresponding to native contacts (Table III), we find-not unexpectedly-that FRET pairs probing the contacts within the same domain are correlated considerably (e.g., for pairs $C_{1}$ and $C_{2}$, or $C_{3}$, and $C_{5}$ ) while the correlation is low when each FRET pair resides on a different domain (e.g., $C_{2}$, and $C_{4}$ ). Interestingly, if one of the FRET pairs probes contact $C_{6}(\{15,45\})$, it shows significant correlation with the other pair regardless of its location.

Returning to Table IV, we find weak structural coupling between two FRET pairs when they are situated on different domains, which is manifested by $\eta_{12}$ values that are one to three orders of magnitude lower than the diagonal terms $\eta_{11}$. This is different from the case of structure 1 , where $\eta_{12}$ tend to be of the same order of magnitude as $\eta_{11}$, the difference never exceeding a factor of $\sim 10$.

\section{CONCLUDING REMARKS}

We have shown here that an analysis of temporal correlations between two FRET signals can provide useful information about structural coupling and cooperativity among various structural elements of a protein. The four-color FRET experiment envisioned here involves two independent donor-acceptor pairs. As discussed in the Introduction, in practice it may be difficult to avoid spectral overlap among

TABLE IV. $\eta_{12}$ for structure 2 where $E^{(1)}$ and $E^{(2)}$ are for FRET pairs attached to the monomers of the corresponding row and column contacts of the table element.

\begin{tabular}{lcccccc}
\hline \hline & $C_{1}$ & $C_{2}$ & $C_{3}$ & $C_{4}$ & $C_{5}$ & $C_{6}$ \\
\hline$C_{1}$ & $-1.3 \times 10^{-3}$ & $-6.3 \times 10^{-4}$ & $-6.8 \times 10^{-5}$ & $-3.3 \times 10^{-6}$ & $-1.6 \times 10^{-5}$ & $-5.0 \times 10^{-4}$ \\
$C_{2}$ & & $-7.2 \times 10^{-4}$ & $-2.3 \times 10^{-5}$ & $-9.7 \times 10^{-7}$ & $-4.9 \times 10^{-6}$ & $-2.2 \times 10^{-4}$ \\
$C_{3}$ & & & $-6.1 \times 10^{-4}$ & $-4.9 \times 10^{-5}$ & $-2.2 \times 10^{-4}$ & $-5.9 \times 10^{-4}$ \\
$C_{4}$ & & & $-8.1 \times 10^{-4}$ & $-3.4 \times 10^{-4}$ & $-3.1 \times 10^{-5}$ \\
$C_{5}$ & & & & $-6.6 \times 10^{-4}$ & $-1.5 \times 10^{-4}$ \\
$C_{6}$ & & & & & $-1.2 \times 10^{-3}$ \\
\hline \hline
\end{tabular}


various dyes. Designing a setup with one donor and two acceptors should be much easier and, in fact, has already been accomplished. ${ }^{36,37}$ Unfortunately, extracting the inherent coupling between the conformational variables of interest from the FRET data becomes much more difficult for such three-color FRET experiments because of the additional coupling that arises due to competition between different FRET pathways. Suppose, for example, that we have one donor D and two acceptors, $A_{1}$ and $A_{2}$, and that only two FRET pathways are possible, $\mathrm{D} \rightarrow \mathrm{A}_{1}$ or $\mathrm{D} \rightarrow \mathrm{A}_{2}$. In the case of weak excitation, the fractions of photons received from each acceptor are then given by ${ }^{37}$

$$
\begin{aligned}
& E^{(1)}=\frac{k_{\mathrm{FRET}}^{(1)}\left(R^{(1)}\right)}{\Gamma+k_{\mathrm{FRET}}^{(1)}\left(R^{(1)}\right)+k_{\mathrm{FRET}}^{(2)}\left(R^{(2)}\right)}, \\
& E^{(2)}=\frac{k_{\mathrm{FRET}}^{(2)}\left(R^{(2)}\right)}{\Gamma+k_{\mathrm{FRET}}^{(1)}\left(R^{(1)}\right)+k_{\mathrm{FRET}}^{(2)}\left(R^{(2)}\right)} .
\end{aligned}
$$

Here $\Gamma^{-1}$ is the radiative lifetime for the donor and $k_{\mathrm{FRET}}^{(1,2)}$ $\left(R^{(1,2)}\right)$ is the FRET rate for each pathway, which depends on the distance between the donor and the respective acceptor. This shows that the emission signal in a given channel will depend on both distances even if the distances $R^{(1)}$ and $R^{(2)}$ themselves are statistically independent. To eliminate this pathway coupling, it is tempting to simply invert Eq. (38), $\left\{E^{(1)}, E^{(2)}\right\} \rightarrow\left\{R^{(1)}, R^{(2)}\right\}$, and use $R^{(1)}$ and $R^{(2)}$ as underlying dynamic variables. The theory of Sec. II would then be directly applied to $R^{(1)}$ and $R^{(2)}$ rather than to $E^{(1)}$ and $E^{(2)}$. The problem with this is that one measures not the instantaneous value of the FRET efficiency but rather an average value corresponding to a given conformational state. ${ }^{41,47}$ This means that Eq. (38) should be replaced by

$$
\begin{aligned}
& E_{i}^{(1)}=\left\langle\frac{k_{\mathrm{FRET}}^{(1)}\left(R^{(1)}\right)}{\Gamma+k_{\mathrm{FRET}}^{(1)}\left(R^{(1)}\right)+k_{\mathrm{FRET}}^{(2)}\left(R^{(2)}\right)}\right\rangle_{\left\{R^{(1)}, R^{(2)}\right\}_{i}}, \\
& E_{i}^{(2)}=\left\langle\frac{k_{\mathrm{FRET}}^{(2)}\left(R^{(2)}\right)}{\Gamma+k_{\mathrm{FRET}}^{(1)}\left(R^{(1)}\right)+k_{\mathrm{FRET}}^{(2)}\left(R^{(2)}\right)}\right\rangle_{\left\{R^{(1)}, R^{(2)}\right\}_{i}},
\end{aligned}
$$

where the subscript $\left\{R^{(1)}, R^{(2)}\right\}_{i}$ denotes averaging over the joint probability distribution $p_{i}\left(R^{(1)}, R^{(2)}\right)$ of $R^{(1)}$ and $R^{(2)}$ corresponding to the conformational state $i$. For the model studied in Sec. III, it is easy to perform the above averaging, because for each set of contacts the joint distribution of the two distances is straightforward to calculate. Going backward and obtaining the joint distributions of distances from $E_{i}^{(\alpha)}$ would be a much more challenging exercise in statistical analysis.

One example where this can be accomplished with relative ease is the case where each conformation $i$ has a well defined geometry so that the distribution $p_{i}\left(R^{(1)}, R^{(2)}\right)$ is sharply peaked at $R^{(1)}=R_{i}^{(1)}, R^{(2)}=R_{i}^{(2)}$. Then Eq. (39) can be approximated by

$$
E_{i}^{(\alpha)}=\frac{k_{\mathrm{FRET}}^{(\alpha)}\left(R_{i}^{(\alpha)}\right)}{\Gamma+k_{\mathrm{FRET}}^{(\alpha)}\left(R_{i}^{(1)}\right)+k_{\mathrm{FRET}}^{(2)}\left(R_{i}^{(2)}\right)}, \quad \alpha=1,2,
$$

and so now one can solve for $R^{(1)}$ and $R^{(2)}$ in each state $i$.
It is likely that future development of multidimensional FRET schemes will require a compromise between the experimental realities of spectral overlap, which make it difficult to avoid competing FRET pathways, and the theoretical models which will be required to use approximations to go beyond the perfect four-color FRET case considered here.

\section{ACKNOWLEDGMENTS}

We are grateful to Rick Russell, Ben Schuler, and Haw Yang, for helpful discussions. This work was supported by the National Science Foundation (Grant No. CHE 0347862) and the Robert A. Welch Foundation (Grant No. F-1514).

${ }^{1}$ S. Weiss, Science 283, 1676 (1999).

${ }^{2}$ X. Zhuang and M. Rief, Curr. Opin. Struct. Biol. 13, 88 (2003).

${ }^{3}$ W. Min, B. P. English, G. Luo, B. J. Cherayil, S. C. Kou, and X. S. Xie, Acc. Chem. Res. 38, 923 (2005).

${ }^{4}$ W. E. Moerner, Proc. Natl. Acad. Sci. U.S.A. 104, 12596 (2007).

${ }^{5}$ P. V. Cornish and T. Ha, ACS Chem. Biol. 2, 53 (2007).

${ }^{6}$ B. Chan, K. Weidemaier, W.-T. Yip, P. F. Barbara, and K. MusierForsyth, Proc. Natl. Acad. Sci. U.S.A. 96, 459 (1999).

${ }^{7}$ A. A. Deniz, M. Dahan, J. R. Grunwell, T. Ha, A. E. Faulhaber, D. S. Chemla, S. Weiss, and P. G. Schultz, Proc. Natl. Acad. Sci. U.S.A. 96, 3670 (1999).

${ }^{8}$ A. A. Deniz, T. A. Laurence, G. S. Beligere, M. Dahan, A. B. Martin, D. S. Chemla, P. E. Dawson, P. G. Schultz, and S. Weiss, Proc. Natl. Acad. Sci. U.S.A. 97, 5179 (2000).

${ }^{9}$ A. A. Deniz, T. A. Laurence, M. Dahan, D. S. Chemla, P. G. Schultz, and S. Weiss, Annu. Rev. Phys. Chem. 52, 233 (2001).

${ }^{10}$ Y. Jia, D. S. Talaga, W. L. Lau, H. S. M. Lu, W. F. DeGrado, and R. M. Hochstrasser, Chem. Phys. 247, 747 (1999).

${ }^{11}$ E. V. Kuzmenkina, C. D. Heyes, and G. U. Nienhaus, Proc. Natl. Acad. Sci. U.S.A. 102, 15471 (2005)

${ }^{12}$ G. Cosa, Y. Zeng, H. W. Liu, C. F. Landes, D. E. Makarov, K. MusierForsyth, and P. F. Barbara, J. Phys. Chem. B 110, 2419 (2006).

${ }^{13}$ J. A. Hanson, K. Duderstadt, L. P. Watkins, S. Bhattacharyya, J. Brokaw, J. W. Chu, and H. Yang, Proc. Natl. Acad. Sci. U.S.A. 104, 18055 (2007).

${ }^{14}$ C. Bai, C. Wang, X. S. Xie, and P. G. Wolynes, Proc. Natl. Acad. Sci. U.S.A. 96, 11075 (1999).

${ }^{15}$ J. Wang and P. Wolynes, Phys. Rev. Lett. 74, 4317 (1995).

${ }^{16}$ Y. Jung, E. Barkai, and R. J. Silbey, J. Chem. Phys. 117, 10980 (2002).

${ }^{17}$ Y. Jung, E. Barkai, and R. J. Silbey, Adv. Chem. Phys. 123, 199 (2002).

${ }^{18}$ J. B. Witkoskie and J. Cao, J. Chem. Phys. 121, 6373 (2004).

${ }^{19}$ J. B. Witkoskie and J. Cao, J. Chem. Phys. 121, 6361 (2004).

${ }^{20}$ O. Flomenbom and R. J. Silbey, Proc. Natl. Acad. Sci. U.S.A. 103, 10907 (2006)

${ }^{21}$ I. V. Gopich and A. Szabo, J. Chem. Phys. 122, 14707 (2005).

${ }^{22}$ I. V. Gopich and A. Szabo, J. Chem. Phys. 124, 154712 (2006).

${ }^{23}$ S. A. McKinney, C. Joo, and T. Ha, Biophys. J. 91, 1941 (2006).

${ }^{24}$ J. Shang and E. Geva, J. Phys. Chem. B 111, 4220 (2007).

${ }^{25}$ J. B. Witkoskie and J. Cao, J. Phys. Chem. B 110, 19009 (2006).

${ }^{26}$ L. P. Watkins and H. Yang, J. Phys. Chem. B 109, 617 (2005).

${ }^{27}$ L. P. Watkins and H. Yang, Biophys. J. 86, 4015 (2004).

${ }^{28}$ F. L. Brown, Acc. Chem. Res. 39, 363 (2006).

${ }^{29}$ F. L. Brown, Phys. Rev. Lett. 90, 028302 (2003).

${ }^{30}$ Y. Peng, Y. Zheng, and F. L. Brown, J. Chem. Phys. 126, 104303 (2007).

${ }^{31}$ Y. Zheng and F. L. Brown, J. Chem. Phys. 121, 3238 (2004).

${ }^{32}$ Y. Zheng and F. L. Brown, Phys. Rev. Lett. 90, 238305 (2003).

${ }^{33}$ A. Gaiduk, R. Kuhnemuth, S. Felekyan, M. Antonik, W. Becker, V. Kudryavtsev, C. Sandhagen, and C. A. Seidel, Microsc. Res. Tech. 70, 433 (2007).

${ }^{34}$ V. Kudryavtsev, S. Felekyan, A. K. Wozniak, M. Konig, C. Sandhagen, R. Kuhnemuth, C. A. Seidel, and F. Oesterhelt, Anal. Bioanal. Chem. 387, 71 (2007).

${ }^{35}$ M. Heilemann, P. Tinnefeld, G. Sanehez Mosteiro, M. Garcia Parajo, N. F. Van Hulst, and M. Sauer, J. Am. Chem. Soc. 126, 6514 (2004).

${ }^{36}$ S. Hohng, C. Joo, and T. Ha, Biophys. J. 87, 1328 (2004).

${ }^{37}$ J. P. Clamme and A. A. Deniz, ChemPhysChem 6, 74 (2005).

${ }^{38}$ M. Heilemann, R. Kasper, P. Tinnefeld, and M. Sauer, J. Am. Chem. Soc. 
128, 16864 (2006).

${ }^{39}$ N. K. Lee, A. N. Kapanidis, H. R. Koh, Y. Korlann, S. O. Ho, Y. Kim, N. Gassman, S. K. Kim, and S. Weiss, Biophys. J. 92, 303 (2007).

${ }^{40}$ X. Zhuang, L. E. Bartley, H. P. Babcock, R. Russell, T. Ha, D. Herschlag, and S. Chu, Science 288, 2048 (2000).

${ }^{41}$ B. Schuler, E. A. Lipman, and W. A. Eaton, Nature (London) 419, 743 (2002).

${ }^{42}$ B. Schuler, E. A. Lipman, P. J. Steinbach, M. Kumke, and W. A. Eaton, Proc. Natl. Acad. Sci. U.S.A. 102, 2754 (2005).

${ }^{43}$ E. Sherman and G. Haran, Proc. Natl. Acad. Sci. U.S.A. 103, 11539 (2006)

${ }^{44}$ D. S. Talaga, W. L. Lau, H. Roder, J. Tang, Y. Jia, W. F. DeGrado, and R. M. Hochstrasser, Proc. Natl. Acad. Sci. U.S.A. 97, 13021 (2000).

${ }^{45}$ S. Weiss, Nat. Struct. Biol. 7, 724 (2000).

${ }^{46}$ E. R. McCarney, J. H. Werner, S. L. Bernstein, I. Ruczinski, D. E. Makarov, P. M. Goodwin, and K. W. Plaxco, J. Mol. Biol. 352, 672 (2005).

${ }^{47}$ D. Nettels, I. V. Gopich, A. Hoffmann, and B. Schuler, Proc. Natl. Acad. Sci. U.S.A. 104, 2655 (2007)

${ }^{48}$ H. Yang, G. Luo, P. Karnchanaphanurach, T. M. Louie, I. Rech, S. Cova, L. Xun, and X. S. Xie, Science 302, 262 (2003).

${ }^{49}$ A. J. Berglund, A. C. Doherty, and H. Mabuchi, Phys. Rev. Lett. 89, 068101 (2002).

${ }^{50}$ Z. S. Wang and D. E. Makarov, J. Phys. Chem. B 107, 5617 (2003).

${ }^{51}$ R. L. Baldwin and G. D. Rose, Trends Biochem. Sci. 24, 77 (1999).

${ }^{52}$ H. S. Chan, S. Bromberg, and K. A. Dill, Philos. Trans. R. Soc. London, Ser. B 348, 61 (1995).

${ }^{53}$ K. A. Dill, K. M. Fiebig, and H. S. Chan, Proc. Natl. Acad. Sci. U.S.A. 90, 1942 (1993).

${ }^{54}$ D. A. Debe, M. J. Carlson, and W. A. Goddard, Proc. Natl. Acad. Sci. U.S.A. 96, 2596 (1999)

${ }^{55}$ D. A. Debe and W. A. Goddard, J. Mol. Biol. 294, 619 (1999).

${ }^{56}$ O. V. Galzitskaya and A. V. Finkelstein, Proc. Natl. Acad. Sci. U.S.A. 96 11299 (1999).

${ }^{57}$ S. A. Islam, M. Karplus, and D. L. Weaver, J. Mol. Biol. 318, 199 (2002).
${ }^{58}$ M. Karplus and D. L. Weaver, Biopolymers 18, 1421 (1979).

${ }^{59}$ D. E. Makarov and K. W. Plaxco, Protein Sci. 12, 17 (2003).

${ }^{60}$ V. Munoz and W. A. Eaton, Proc. Natl. Acad. Sci. U.S.A. 96, 11311 (1999).

${ }^{61}$ J. K. Myers and T. G. Oas, Nat. Struct. Biol. 8, 552 (2001).

${ }^{62}$ G. D. Rose, J. Mol. Biol. 134, 447 (1979).

${ }^{63}$ Y. Q. Zhou and M. Karplus, Nature (London) 401, 400 (1999).

${ }^{64}$ J. M. Fernandez and H. Li, Science 303, 1674 (2004).

${ }^{65}$ R. B. Best and G. Hummer, Science 308, 498 (2005).

${ }^{66}$ S. Kirmizialtin, L. Huang, and D. E. Makarov, J. Chem. Phys. 122, 234915 (2005).

${ }^{67}$ X. Qi and J. J. Portman, Proc. Natl. Acad. Sci. U.S.A. 104, 10841 (2007).

${ }^{68}$ M. S. Li, D. K. Klimov, and D. Thirumalai, Phys. Rev. Lett. 93, 268107 (2004).

${ }^{69}$ T. R. Weikl, M. Palassini, and K. A. Dill, Protein Sci. 13, 822 (2004).

${ }^{70}$ P. Bruscolini, A. Pelizzola, and M. Zamparo, J. Chem. Phys. 126, 215103 (2007).

${ }^{71}$ J. D. Chodera, N. Singhal, and V. S. Pande, K. A. Dill, and W. C. Swope, J. Chem. Phys. 126, 155101 (2007).

${ }^{72}$ D. E. Makarov, C. A. Keller, K. W. Plaxco, and H. Metiu, Proc. Natl. Acad. Sci. U.S.A. 99, 3535 (2002).

${ }^{73}$ D. E. Makarov and H. Metiu, J. Chem. Phys. 116, 5205 (2002).

${ }^{74}$ K. Eom, P.-C. Li, D. E. Makarov, and G. J. Rodin, J. Phys. Chem. B 107, $8730(2003)$

${ }^{75}$ K. Eom, D. E. Makarov, and G. J. Rodin, Phys. Rev. E 71, 021904 (2005).

${ }^{76}$ N. G. van Kampen, Stochastic Processes in Physics and Chemistry (Elsevier, New York/North Holland, Amsterdam, 1981).

${ }^{77}$ K. W. Plaxco, K. T. Simons, I. Ruczinski, and D. Baker, Biochemistry 39, 11177 (2000).

${ }^{78}$ D. E. Makarov and G. J. Rodin, Phys. Rev. E 66, 011908 (2002).

${ }^{79}$ A. Szabo, K. Schulten, and Z. Schulten, J. Chem. Phys. 72, 4350 (1980).

${ }^{80}$ I. V. Gopich and A. Szabo, J. Phys. Chem. B 107, 5058 (2003).

${ }^{81}$ K. W. Plaxco, K. T. Simons, and D. Baker, J. Mol. Biol. 277, 985 (1998).

${ }^{82}$ H. S. Chan and K. A. Dill, Proteins 30, 2 (1998). 